\title{
Primary orbital amyloidosis localised to an extraocular muscle
}

\author{
GERD E HOLMSTRÖM AND KARL G NYMAN \\ From the Department of Ophthalmology, Danderyds Hospital, Stockholm, Sweden
}

SUMMARY Primary, localised orbital amyloidosis has been reported previously in only 15 patients. A case with amyloidosis strictly localised to an extraocular muscle is presented.

Ophthalmic involvement frequently occurs in generalised amyloidosis. ${ }^{12}$ Localised orbital amyloidosis is very rare and has been reported in only 15 patients. A patient with amyloidosis strictly localised to an extraocular muscle is presented. This has not been previously reported.

\section{Case report}

For the last 10 years a 60 -year-old woman had a tendency to diplopia when looking quickly to one side. In 1984 a clinical examination revealed a right exotropia, and a Lees screen showed reduced motility of her right eye, particularly a reduction of the adduction - that is, underaction of the medial rectus muscle. There was also a strong overaction of the lateral rectus muscle of the left eye. A forced Correspondence to G E Holmström, Department of Ophthalmology, Danderyds Hospital, S-182 88 Danderyd, Sweden.

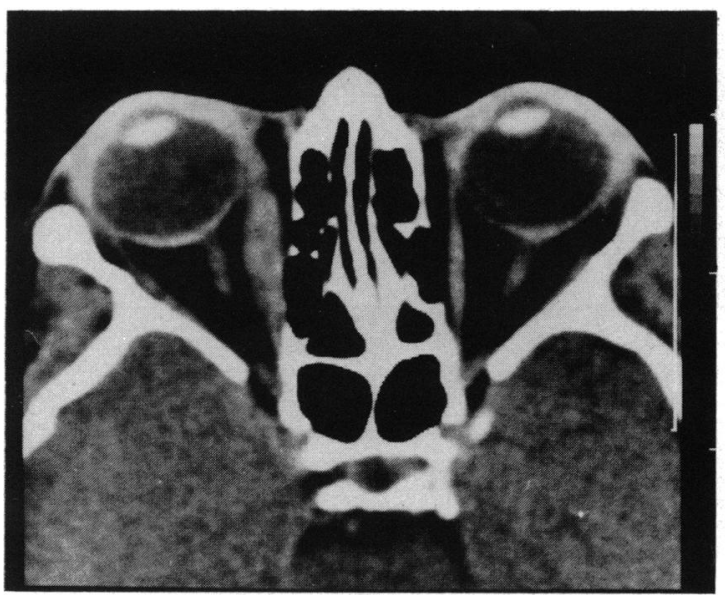

Fig. 1 CT scanning of the orbits, $4 \mathrm{~mm}$ thick section parallel with the optic nerves. duction test of the right eye revealed great resistance in both adduction and abduction. The patient had normal visual acuity and only a slight hypermetropia $(+0.5$ right and left eyes). Her fundi were normal and no visual field defects were noticed. Computed tomographic (CT) scanning showed a normal brain configuration, but in the right orbit the medial rectus muscle had a fusiform thickening, being three times as thick as the left medial rectus muscle (Figs. 1,2). No infiltration or destruction of the surrounding structures was seen.

In January 1985 the patient underwent surgery for her right exotropia (resection of the right medial rectus muscle and recession of the right lateral rectus muscle, with a traction suture in adduction). A biopsy of her right medial rectus muscle was also

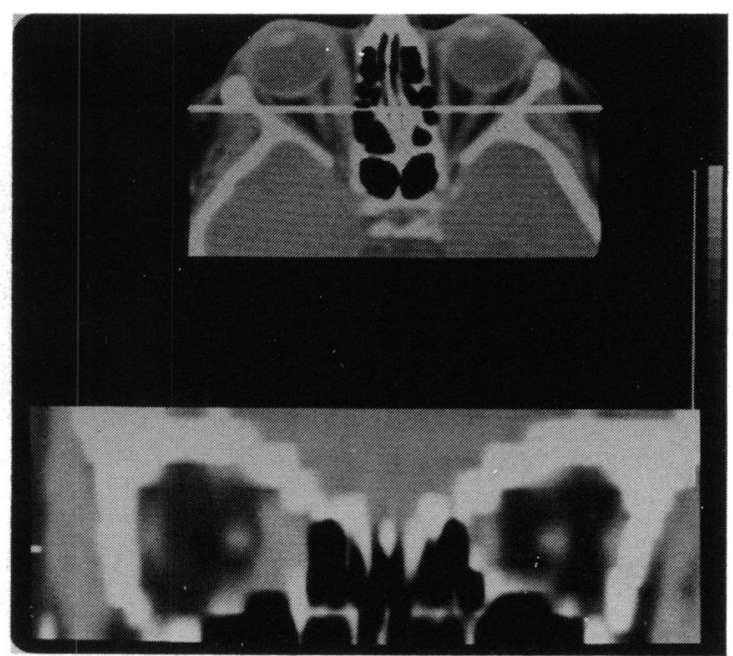

Fig. 2 Reconstruction of sections retrobulbarly through the orbits, demonstrating the optic nerves and the extraocular muscles in frontal projection. 
performed. This muscle appeared waxy and showed ordinary muscle structure only in the $2 \mathrm{~mm}$ closest to its insertion. Pathological examination revealed an amorphous structure which, after staining with Congo red, showed a green birefringence. Only a few fragments of the ordinary muscle structure could be identified. The histopathological diagnosis was amyloidosis.

A pulmonary $x$-ray was normal. Phonocardiography and ultracardiography did not show any abnormality. Biopsies of the subcutaneous fat from the abdomen and the cheek showed no amyloid deposits. No proteinuria was found, and the results of serum and urine electrophoresis were normal. No rheumatoid factor or antibodies against cell nuclei, smooth muscle, glomeruli, and mitochondria could be found. There was therefore no sign of generalised amyloidosis.

In April 1985 the patient underwent supplementary surgery for a slight esotropia with intermittent diplopia (recession of the right medial rectus muscle with an adjustable suture). A CT scan in July 1985 showed the same picture as before.

The patient will be followed up with ophthalmic examinations, tests of visual fields, and CT scanning.

\section{Discussion}

Amyloid is an extracellular, proteinaceous material that can be deposited in various tissues. Histopathological identification is best made with Congo red staining. When viewed with a polarising microscope all forms of amyloid show a green birefringence.

Amyloidosis is the term for a heterogeneous group of diseases characterised by accumulation of amyloid in different tissues. It has been subjected to many classifications. A clinically widely used classification is: (1) primary generalised amyloidosis (without any underlying disease); (2) secondary amyloidosis (associated with, for example, chronic infection, rheumatoid arthritis); (3) amyloidosis associated with myelomatosis; and (4) tumour-forming amyloidosis, characterised by localised amyloid masses. ${ }^{3}$ Some authors also note a fifth, hereditary, form of amyloidosis. ${ }^{4}$ Analyses of the chemical structure of amyloid are now making new classifications possible. ${ }^{5}$

Ophthalmic involvement has been reported in all subgroups of amyloidosis described above. ${ }^{4}$ The case presented here is of localised orbital amyloidosis. Apart from amyloid tumours of the conjunctiva and lid, localised orbital amyloidosis is rare, ${ }^{2}$ and only 15 patients with primary localised orbital amyloidosis have been reported so far. ${ }^{1246-16}$ Four of the previously reported 15 cases were bilateral. Three patients had their lesions localised in the lacrimal gland. In most of the remaining patients the amyloid tumour extended deep and infiltrated the orbit. No case has previously been reported in which the amyloid tumour is localised only to an extraocular muscle.

In conclusion, localised orbital amyloidosis seems to be rare. This is the first case reported with amyloid entirely localised to an extraocular muscle.

Dr A Moberg, Department of Pathology, Danderyds Hospital, kindly helped with the histopathological investigation.

\section{References}

1 Groniowski J, Bernardczykowa A, Norn MS. Orbital amyloidosis. Acta Ophthalmol (Kbh) 1965; 43: 725-9.

2 Howard GM. Amyloid tumours of the orbit. Br J Ophthalmol 1966; 50: 421-5.

3 Reimann HA, Koucky RF, Eklund CM. Primary amyloidosis limited to tissue of mesodermal origin. Am J Pathol 1935; 11: 977-88.

4 Knowles DM, Jakobiec FA, Rosen M, Howard G. Amyloidosis of the orbit and adnexae. Surv Ophthalmol 1975; 19: 367-84.

5 Glenner GG. Amyloid deposits and amyloidosis. The $\beta$ fibrilloses. N Engl J Med 1980; 302: 1283-92, 1333-43.

6 Pollems W. Über tumorförmige lokale Amyloidosis in der Orbita. Graefes Arch Clin Exp Ophthalmol 1920; 101: 346-61.

7 Handousa A. Localised intra-orbital amyloid disease. $\mathrm{Br} J$ Ophthalmol 1954; 38: 510-1.

8 Easton JA, Smith WT. Non-specific granuloma of the orbit ('orbital pseudotumor'). J Pathol Bacteriol 1961; 82: 345-54.

9 Kassman T, Sundmark E. Orbital pseudo-tumours with amyloid. Acta Ophthalmol (Kbh) 1967; 45: 220-8.

10 Raab EL. Intraorbital amyloid. Br J Ophthalmol 1970; 54: 445-9.

11 Radnot M, Lapis K, Feher K. Amyloid tumour in the lacrimal gland. Ann Ophthalmol 1971; 3: 727-42.

12 Schubert E. Amyloidtumor der Orbita. Klin Monatsbl Augenheilkd 1972; 160: 467-8.

13 Savino PJ, Schatz NJ, Rodrigues MM. Orbital amyloidosis. Can J Ophthalmol 1976; 11: 252-5.

14 Jensen OA. Bilateral amyloidosis of the orbit. Ophthalmologica 1976; 173: 70-8.

15 Nehen JH. Primary localised orbital amyloidosis. Acta Ophthalmol (Kbh) 1979; 57: 287-95.

16 Cohen MM, Lessell S. Amyloid tumor of the orbit. Neuroradiology, 1979; 18: 157-9.

Accepted for publication 3 April 1986. 